\section{Osteolytic Lesion in PAPA Syndrome Responding to Anti-interleukin 1 Treatment}

\section{To the Editor:}

Born from an uneventful pregnancy and in complete well-being, a girl at the age of 18 months presented arthritis of the left knee associated to low-grade fever, leukocytosis, and increased inflammatory markers. The arthrocentesis revealed turbid fluid with a negative culture. The girl was treated with intravenous antibiotic therapy with a temporary partial improvement, but pain and swelling of the left knee recurred and worsened in the subsequent months. Pain at the lower left limb was also referred without signs of arthritis at the ankle. Radiograph of the lower limbs was performed revealing the presence of an osteolytic area of about $2.3 \mathrm{~cm} \times$ $0.7 \mathrm{~cm}$ with periosteal apposition in the distal diaphysis of the left tibia. Bone scintigraphy showed a metabolic hyperactivity in the same area only. Bone biopsy was negative for malignancy, histiocytosis, or tuberculosis while revealing the presence of an inflammatory infiltrate with neutrophils, macrophages, and monocytes, consistent with a diagnosis of chronic osteomyelitis. Nonsteroidal antiinflammatory drug treatment was started, with poor response. Bone marrow aspiration was negative for malignancies. A diagnosis of chronic recurrent multifocal osteomyelitis (CRMO) was made; prednisone $1 \mathrm{mg} / \mathrm{kg} /$ day was started with a prompt good response, but the recurrence of bone pain at steroid discontinuation.

At 33 months, the girl was referred to our center. The blood test revealed a slight neutrophilic leukocytosis with elevation of acute-phase reactants [C-reactive proteins (CRP) $4.28 \mathrm{mg} / \mathrm{dl}$, erythrocyte sedimentation rate $39 \mathrm{~mm} / \mathrm{h}$ ]. The radiograph of the left limb confirmed the presence of the osteolytic lesion with periostitis (Figure 1A), and the whole-body short-tau inversion recovery-magnetic resonance imaging (MRI) revealed a bilateral alteration of signal in the diaphysis and distal metaphysis of the tibia and in the left heel (Figure 1B). In the following weeks, the patient had a relapse of arthritis of the right knee associated with low-grade fever. The right knee ultrasound and MRI revealed the presence of intraarticular effusion with synovial vegetations, and blood tests showed a marked leukocytosis with increase of acute-phase reactants (CRP $9.04 \mathrm{mg} / \mathrm{dl}$, serum amyloid A $987 \mathrm{mg} / \mathrm{l})$. The right knee arthrocentesis revealed a turbid fluid with a very large prevalence of polymorphonuclear cells; cultures were negative. The arthritis completely resolved after an intraarticular injection of triamcinolone hexacetonide.

The careful evaluation of family history revealed the presence of severe acne in the father since adolescence. On this basis, a PAPA (pyogenic arthritis, pyoderma gangrenosum, and acne) syndrome was suspected. The molecular analysis of PSTPIP1 gene revealed the E250Q pathogenic mutation ${ }^{1}$ in both the patient and the father, confirming the final diagnosis.

Treatment with recombinant interleukin 1 (IL-1) receptor antagonist (anakinra) was started with a good control of bone pain and arthritis in the following months. After 6 months, acute-phase reactants normalized and the whole-body MRI was negative for osteolytic bone lesions.

PAPA syndrome (MIM 604416) is an autosomal dominant condition characterized by the classical triad of pyogenic sterile arthritis, pyoderma gangrenosum, and cystic acne ${ }^{2}$. The clinical manifestations of this disease are variable, and as observed in our present case, the penetrance of the mutations in the causative gene, PSTPIP1, might be variable in the context of the same family ${ }^{2,3}$.

The presence of inflammatory osteolytic lesions is not considered a typical feature of the disease. In a study, Demidowich, et al reported the occurrence of an osteomyelitis in 1 patient belonging to a small cohort of patients with PAPA. However, a detailed description of radiological and histological findings was not available ${ }^{3}$.

The PSTPIP1 is a cytoskeletal adaptor protein that interacts with a number of other intracellular or membrane proteins such as PTP-PEST, CD2, WASP, Fas ligand, and pyrin ${ }^{2}$. It has been proposed that the interaction between pyrin and PSTPIP1 is involved in the intracellular mechanisms leading to the activation and secretion of IL- $1 \beta^{4}$. Interestingly, recessive mutations of PSTPIP2 gene, a homolog of PSTPIP1, cause a phenotype characterized by multifocal osteolytic lesions in a murine model of the disease ${ }^{5}$.

Sterile osteolytic bone lesions are a common finding of autoinflammatory diseases: the monogenic Majeed and DIRA (deficiency of IL-1

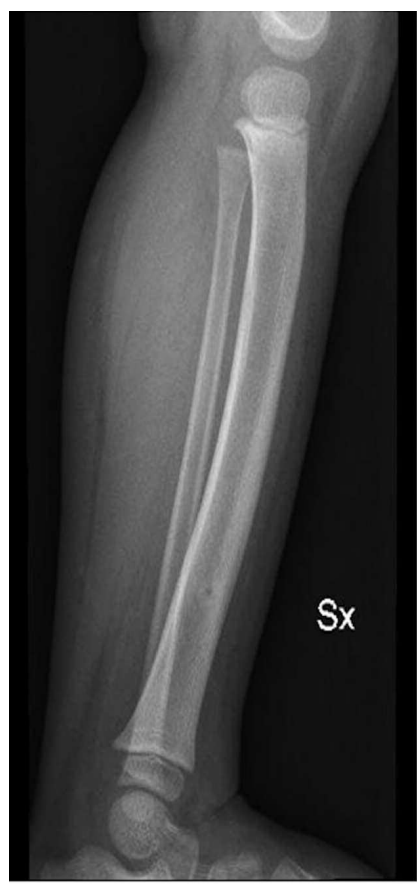

$1 \mathrm{~A}$

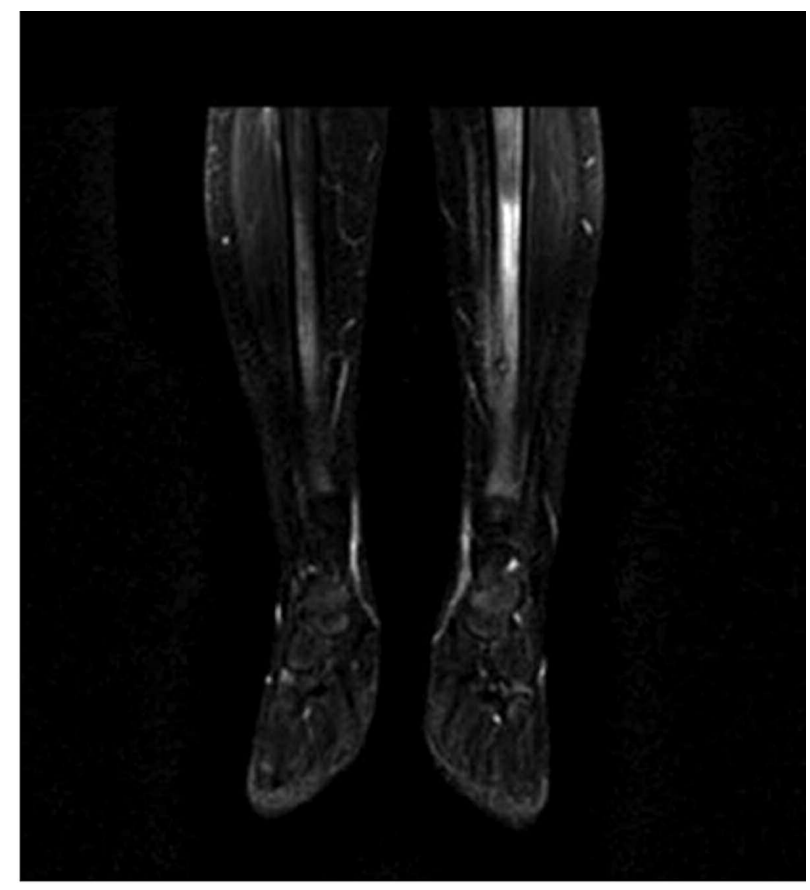

$1 \mathrm{~B}$

Figure 1. Bone lesion on the left tibia revealed by radiograph (1A) and magnetic resonance imaging (1B). 
receptor antagonist) syndromes, and the multifactorial $\mathrm{CRMO}^{6,7,8}$. A good response to IL-1 blockers has been reported in all these conditions $7,8,9$.

The association of CRMO with arthritis and pyogenic skin manifestations identifies a distinct clinical entity called SAPHO (synovitis, acne, pustulosis, hyperostosis, and osteitis) syndrome, usually observed in young adults and characterized, at least in few anecdotal cases, by the same positive response to anti-IL- 1 treatment ${ }^{10}$.

Our present case enlarges the clinical spectrum of the manifestations possibly associated to PAPA syndrome. It also suggests that the molecular analysis of PSTPIP1 gene should be considered in all patients presenting with bone lesions associated with arthritis and/or pyogenic skin manifestations.

The positive response to anti-IL-1 treatment observed in this patient strengthens the evidence for a pivotal role of IL-1 in the pathogenesis of autoinflammatory diseases characterized by inflammatory osteolytic bone lesions ${ }^{8}$.

\section{ROBERTA CAORSI, MD; PAOLO PICCO, MD; ANTONELLA} BUONCOMPAGNI, MD; ALBERTO MARTINI, Professor; MARCO GATTORNO, MD, Unità Operativa Pediatria II, Giannina Gaslini Institute; Department of Pediatrics, University of Genoa, Genoa, Italy.

Address correspondence to Dr. Marco Gattorno, MD, UO Pediatria 2, Istituto Giannina Gaslini, Largo Giannina Gaslini 5, 16147, Genoa, Italy. E-mail: marcogattorno@ospedale-gaslini.ge.it

\section{REFERENCES}

1. Wise CA, Gillum JD, Seidman CE, Lindor NM, Veile R, Bashiardes S, et al. Mutations in CD2BP1 disrupt binding to PTP PEST and are responsible for PAPA syndrome, an autoinflammatory disorder. Hum Mol Genet 2002;11:961-9.

2. Smith EJ, Allantaz F, Bennett L, Zhang D, Gao X, Wood G, et al. Clinical, molecular, and genetic characteristics of PAPA syndrome: a review. Curr Genomics 2010;11:519-27.
3. Demidowich AP, Freeman AF, Kuhns DB, Aksentijevich I, Gallin JI, Turner ML, et al. Brief report: genotype, phenotype, and clinical course in five patients with PAPA syndrome (pyogenic sterile arthritis, pyoderma gangrenosum, and acne). Arthritis Rheum 2012;64:2022-7.

4. Shoham NG, Centola M, Mansfield E, Hull KM, Wood G, Wise $\mathrm{CA}$, et al. Pyrin binds the PSTPIP1/CD2BP1 protein, defining familial Mediterranean fever and PAPA syndrome as disorders in the same pathway. Proc Natl Acad Sci USA 2003;100:13501-6.

5. Ferguson PJ, Bing X, Vasef MA, Ochoa LA, Mahgoub A, Waldschmidt TJ, et al. A missense mutation in pstpip2 is associated with the murine autoinflammatory disorder chronic multifocal osteomyelitis. Bone 2006;38:41-7.

6. Ferguson PJ, Chen S, Tayeh MK, Ochoa L, Leal SM, Pelet A, et al. Homozygous mutations in LPIN2 are responsible for the syndrome of chronic recurrent multifocal osteomyelitis and congenital dyserythropoietic anaemia (Majeed syndrome). J Med Genet 2005;42:551-7.

7. Aksentijevich I, Masters SL, Ferguson PJ, Dancey P, Frenkel J, van Royen-Kerkhoff A, et al. An autoinflammatory disease with deficiency of the interleukin-1-receptor antagonist. N Engl J Med 2009;360:2426-37.

8. Ferguson PJ, Sandu M. Current understanding of the pathogenesis and management of chronic recurrent multifocal osteomyelitis. Curr Rheumatol Rep 2012;14:130-41.

9. Herlin T, Fiirgaard B, Bjerre M, Kerndrup G, Hasle H, Bing X, et al. Efficacy of anti-IL-1 treatment in Majeed syndrome. Ann Rheum Dis 2013;72:410-3.

10. Nguyen MT, Borchers A, Selmi C, Naguwa SM, Cheema G, Gershwin ME. The SAPHO syndrome. Semin Arthritis Rheum 2012;42:254-65.

J Rheumatol 2014;41:11; doi:10.3899/jrheum.140060 